\title{
Why is the Speed of Light in Vacuum a Universal Physical Constant?
}

\author{
Kennard Callender \\ Universidad Tecnológica de Panamá \\ Centro Regional de Veraguas
}

\begin{abstract}
An interpretation of the Lorentz transformation is formulated to answer the question regarding the constancy of the speed of light. It is shown that the observed speed of light in vacuum is a universal constant because physical space is a four-dimensional incompressible inviscid fluid.
\end{abstract}

\section{CONTENTS}

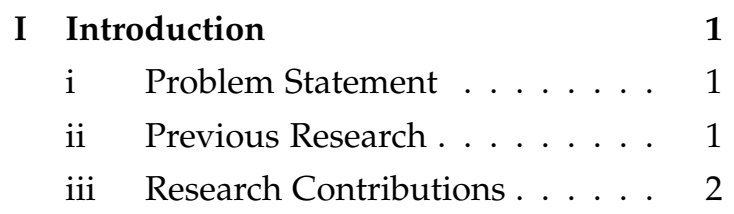

III Conclusion

\section{INTRODUCTION}

$\mathrm{T}$ HE Lorentz transformation was developed to explain why the observed speed of light in vacuum is independent of the frame of reference. Albert Einstein decided to elevate the constancy of the speed of light to a postulate and, together with the principle of relativity, derived the Lorentz transformation from it. That result led him to reject Newton's concept of absolute time and to interpret time intervals as dependent on the observer's frame of reference.

\section{i. Problem Statement}

In the present paper, we propose that the speed of light in vacuum is measured to be the same by all non-accelerating observers because physical space is a four-dimensional incompressibe inviscid fluid. To prove this thesis, we derive the Lorentz transformation from the Galilean transformation for four-dimensional frames of references.

ii. Previous Research

In 1914, Gunnar Nordström unified his theory of gravity with electromagnetism by assuming physical space is four-dimensional [1]. A few years later, Theodor Kaluza unified Einstein's general relativity with Maxwell's electromagnetism in a five-dimensional manifold (four dimensions of space and one of time) [2]. These unifications incentivated us to explore the four-dimensional space hypothesis further.

Recently, we formulated an interpretation of quantum mechanics based on the hypothesis that physical space is a four-dimensional incompressible inviscid fluid [3]. In it, we postulated that time is absolute. Einstein's interpretation of the Lorentz transformation rejects that concept. Therefore, we need to prove there is a valid interpretation of the Lorentz transformation that is consistent with the absolute time postulate. 


\section{iii. Research Contributions}

To the best of the author's knowledge, the following are original contributions from the present article:

- Proposing that the constancy of the speed of light is a result of physical space being a four-dimensional incompressible inviscid fluid.

- Deriving the Lorentz transformation from the Galilean transformation for fourdimensional frames of reference.

\section{The LoREntz Transformation}

$\mathrm{I}$ $\mathbf{N}$ this section we propose an interpretation of the Lorentz transformation that explains why the constancy of the speed of light is a result of physical space being a four-dimensional incompressible inviscid fluid. First, we state the postulates our interpretation is based on. Then, we derive the Lorentz transformation from the Galilean transformation for four-dimensional frames of reference. Finally, we interpret the implications of that derivation.

\section{i. Postulates}

The interpretation we propose of the Lorentz transformation is based on the following postulates:

- Time is absolute.

- Physical space is four-dimensional.

- Physical space is an incompressible inviscid fluid.

- Based on their perception at the macroscopic level, observers assume physical space is three-dimensional, and this assumption has unexpected effects on the measurements they perform.

- Elementary particles move at a constant speed relative to physical space.

- Light propagates through physical space at a constant speed.

These postulates are consistent with the postulates from the Four-Dimensional Fluid Space Interpretation of Quantum Mechanics [3].

\section{ii. Derivation}

We proceed to derive the Lorentz transformation from the Galilean transformation for fourdimensional frames of reference. All the variables, parameters, and constants in this derivation are elements of the real numbers. We start by considering two sets of coordinates that describe the position of events taking place in four-dimensional space. These coordinates are related by the following Galilean transformation:

$$
\begin{gathered}
\left(\begin{array}{c}
x^{\prime \prime} \\
w^{\prime \prime}
\end{array}\right)=\left(\begin{array}{c}
x-v_{x} t \\
w-v_{w} t
\end{array}\right) \\
\left(\begin{array}{l}
y^{\prime \prime} \\
z^{\prime \prime} \\
t^{\prime \prime}
\end{array}\right)=\left(\begin{array}{l}
y \\
z \\
t
\end{array}\right)
\end{gathered}
$$

where

$$
c^{2}=v_{x}^{2}+v_{w}^{2}
$$

Then, we find the coordinates from a rotated frame of reference such that

$$
\begin{gathered}
\left(\begin{array}{c}
x^{\prime \prime} \\
w^{\prime \prime}
\end{array}\right)=\left(\begin{array}{cc}
\cos \theta & \sin \theta \\
-\sin \theta & \cos \theta
\end{array}\right)\left(\begin{array}{c}
x^{\prime} \\
w^{\prime}
\end{array}\right) \\
\left(\begin{array}{l}
y^{\prime \prime} \\
z^{\prime \prime} \\
t^{\prime \prime}
\end{array}\right)=\left(\begin{array}{l}
y^{\prime} \\
z^{\prime} \\
t^{\prime}
\end{array}\right)
\end{gathered}
$$

where the angle $\theta$ is specified by

$$
\begin{aligned}
& \sin \theta=\frac{v_{x}}{c} \\
& \cos \theta=\frac{v_{w}}{c}
\end{aligned}
$$

Finally, from these set of equations, we get the transformation between the coordinates of the two four-dimensional frames of reference we are interested in. The Lorentz transformation will be derived from the following equations:

$$
\left(\begin{array}{cc}
\cos \theta & \sin \theta \\
-\sin \theta & \cos \theta
\end{array}\right)\left(\begin{array}{c}
x^{\prime} \\
w^{\prime}
\end{array}\right)=\left(\begin{array}{c}
x-v_{x} t \\
w-v_{w} t
\end{array}\right)
$$

$$
\left(\begin{array}{l}
y^{\prime} \\
z^{\prime} \\
t^{\prime}
\end{array}\right)=\left(\begin{array}{l}
y \\
z \\
t
\end{array}\right)
$$


The fourth postulate listed in section 2.1 has consequences that can be represented by mathematical conditions. These conditions will be stated in this section but formally defined in section 2.3. Two of them are:

$$
\begin{aligned}
& w^{\prime}=0 \\
& v_{x}=v
\end{aligned}
$$

By substituting eqs. 3 and 11 into eqs. 6 and 7 we get

$$
\begin{gathered}
\sin \theta=\frac{v}{c} \\
\cos \theta=\frac{\sqrt{c^{2}-v^{2}}}{c}=\frac{1}{\gamma}
\end{gathered}
$$

where $\gamma$ is the Lorentz factor, defined by

$$
\gamma=\frac{c}{\sqrt{c^{2}-v^{2}}}
$$

Writing eq. 8 as a set of equation gives

$$
\begin{gathered}
x^{\prime} \cos \theta+w^{\prime} \sin \theta=x-v_{x} t \\
-x^{\prime} \sin \theta+w^{\prime} \cos \theta=w-v_{w} t
\end{gathered}
$$

Substituting eqs. 10, 11, and 13 into eq. 15 gives the following result:

$$
\begin{gathered}
\frac{x^{\prime}}{\gamma}=x-v t \\
x^{\prime}=\gamma(x-v t)
\end{gathered}
$$

This is the $x^{\prime}$ coordinate of the Lorentz transformation. A symmetry condition is imposed on that coordinate by the fourth postulate of section 2.1. Therefore we have

$$
x=\gamma\left(x^{\prime}+v t^{\prime}\right)
$$

which is the $x$ coordinate of the Lorentz transformation. Now, this equation is not consistent with eqs. 8 and 9, because it implies

$$
t^{\prime} \neq t
$$

In fact, eqs. 17 and 18 form a set of simultaneous equations that give

$$
t^{\prime}=\gamma\left(t-v x / c^{2}\right)
$$

$$
t=\gamma\left(t^{\prime}+v x^{\prime} / c^{2}\right)
$$

which are the $t^{\prime}$ and $t$ coordinates of the Lorentz transformation. The other coordinates of that transformation are obtained from eq. 9, so we have

$$
\begin{aligned}
& y^{\prime}=y \\
& z^{\prime}=z
\end{aligned}
$$

The $w^{\prime}$ and $w$ coordinates are not part of the Lorentz transformation because the observer assumes physical space only has three spatial dimensions. However, it can be shown that by manipulating eqs. $6,7,10,11,13,16$, and 21 we get

$$
t^{\prime}=\frac{w}{c}
$$

and applying the symmetry condition gives

$$
t=\frac{w^{\prime}}{c}
$$

The time intervals are derived from eqs. 24 and 25. They are given by

$$
\begin{aligned}
& \Delta t^{\prime}=\frac{\Delta w}{c} \\
& \Delta t=\frac{\Delta w^{\prime}}{c}
\end{aligned}
$$

From these equations, we can see where the frame of reference dependence of the time intervals comes from (according to the Lorentz transformation).

\section{iii. Interpretation}

The results obtained from section 2.2 are interpreted here. But first, we point out some relevant consequences of the postulates listed in section 2.1. These consequences are:

- Physical space is a privileged frame of reference because elementary particles and light move at constant speeds with respect to it. 
- Physical space is an incompressible inviscid fluid. Therefore, physical space is the continuous medium (required by mechanical waves) through which electromagnetic and gravitational waves propagate.

- Light travels at a constant speed through physical space, which means the speed of light in vacuum is independent of the velocity of the source's reference frame.

- The fourth postulate of section 2.1, which we call the observer's assumption, points out that one wrong assumption can lead to other wrong assumptions, and those assumptions to the wrong conclusions.

With this in mind, we proceed to interpret the derivation presented in section 2.2. We start with eqs. 8 and 9 . Those equations represent a transformation between the coordinates of two frames of reference in four-dimensional space. That transformation is a composition of a spatial rotation and a Galilean transformation. It accurately represents and describes what is actually happening. However, the wrong assumption about the dimensionality of physical space can lead to other wrong assumptions and to the wrong conclusions.

According to the observer's assumption, two individuals moving relative to each other in the frames of reference described by eqs. 8 and 9 will believe physical space is three-dimensional. Therefore, they will make the following assumptions about the reference frames:

- Physical events take place in threedimensional space.

- The $x^{\prime}$-axis and the $x$-axis of their frames of reference are always colinear.

- Their three-dimensional inertial frames of reference move relative to each other at a constant velocity $v$ along their common $x^{\prime}-x$ axis.

- The transformation of the coordinates of their frames of reference must be symmetric.

These assumptions can be expressed as mathematical conditions. We proceed to explain the two equations and the symmetry condition that result from them.
The first mathematical condition is given by eq. 10, and it is the result of observer's assuming all physical events must take place in threedimensional space. That assumption forces the $w^{\prime}$ coordinate of the position of every event to be equal to zero. Therefore, when a physical event takes place, what the observer is actually detecting is the light arriving from that event to the $x^{\prime}$-axis.

From this we conclude that the events described by the Lorentz transformation correspond to light been detected along the $x^{\prime}$-axis or the $x$-axis. Consequently, the light from an event taking place along the $x^{\prime}$-axis, takes some time to arrive to the measurement devices along the $x$-axis, and this phenomena is the source of the time dilation effect (as can be seen from eqs. 24 through 27).

The second condition, represented by eq. 11, reflects the fact that one of the individuals assumes the velocity along his $x$-axis is the relative velocity between the reference frames. Thus, he sets the relative velocity $v$ to be equal to the velocity along his $x$-axis $v_{x}$. However, the velocity along the $x$-axis is only the $x$ component of the correct velocity between their frames of reference.

The final condition, based on their assumptions, states that the transformation between the coordinates of their reference frames must be symmetric. Therefore, all they have to do to get the other observer's coordinates is to interchange the primed and unprimed terms, and substitute the relative velocity $v$ by $-v$. This wrong assumption is what leads observers to abandon the concept of absolute time.

Finally, the derivation in section 2.2 shows that all the consequences observed from the Lorentz transformation (including the constancy of the speed of light) are the result of physical space being a four-dimensional incompressible inviscid fluid.

\section{Conclusion}

W E formulated an interpretation of the Lorentz transformation to answer the question regarding the constancy of 
the speed of light. The interpretation is based on postulates that are consistent with the postulates of the Four-Dimensional Fluid Space Interpretation of Quantum Mechanics [3]. These postulates were used to derive the Lorentz transformation from the Galilean transformation for four-dimensional frames of reference. The interpretation of those postulates and that derivation show the constancy of the speed of light, and all the consequences observed from the Lorentz transformation, are the result of physical space being a four-dimensional incompressible inviscid fluid.

\section{Dedication}

$7 \Gamma^{\text {HE }}$ research presented here is dedicated to the memory of my father, Dr. Lorenzo

1 León Callender López, who always supported me and was there for me. Without him, this research would not have been possible.

\section{REFERENCES}

[1] Nordström, Gunnar. Uber die Moglichkeit, das Elektromagnetische Feld und das Gravitationsfeld $\mathrm{Zu}$ Vereinigen. (German) [On the Possibility of Unifying the Electromagnetic and the Gravitational Fields]. Phys. Z. 1914; 15: 504-506

[2] Kaluza, Theodor. Zum Unitätsproblem der Physik. (German) [On the Unification Problem in Physics]. Sitzungsberichte der Königlich Preußischen Akademie der Wissenschaften (Berlin). 1921; 1921: 966-972

[3] Callender, Kennard. The Four-dimensional Fluid Space Interpretation of Quantum Mechanics. OSF Preprints. September 10, 2020. doi:10.31219/osf.io/2y3t5. 